\title{
Rethinking the linear regression model for spatial ecological data
}

Published version

Helene H. Wagner

Wagner, H. H. (2013), Rethinking the linear regression model for spatial ecological data. Ecology, 94: 23812391. doi:10.1890/12-1899.1

Copyright by the Ecological Society of America.

\section{HOW TO CITE TSPACE ITEMS}

Always cite the published version, so the author(s) will receive recognition through services that track citation counts, e.g. Scopus. If you need to cite the page number of the TSpace version (original manuscript or accepted manuscript) because you cannot access the published version, then cite the TSpace version in addition to the published version using the permanent URI (handle) found on the record page. 


\title{
Rethinking the linear regression model for spatial ecological data
}

\author{
Helene H. Wagner ${ }^{1}$ \\ Department of Ecology and Evolutionary Biology, University of Toronto, 3359 Mississauga Road, \\ Mississanga, Ontario L5L 1C6 Canada
}

\begin{abstract}
The linear regression model, with its numerous extensions including multivariate ordination, is fundamental to quantitative research in many disciplines. However, spatial or temporal structure in the data may invalidate the regression assumption of independent residuals. Spatial structure at any spatial scale can be modeled flexibly based on a set of uncorrelated component patterns (e.g., Moran's eigenvector maps, MEM) that is derived from the spatial relationships between sampling locations as defined in a spatial weight matrix. Spatial filtering thus addresses spatial autocorrelation in the residuals by adding such component patterns (spatial eigenvectors) as predictors to the regression model. However, space is not an ecologically meaningful predictor, and commonly used tests for selecting significant component patterns do not take into account the specific nature of these variables. This paper proposes "spatial component regression" (SCR) as a new way of integrating the linear regression model with Moran's eigenvector maps. In its unconditioned form, SCR decomposes the relationship between response and predictors by component patterns, whereas conditioned SCR provides an alternative method of spatial filtering, taking into account the statistical properties of component patterns in the design of statistical hypothesis tests. Application to the well-known multivariate mite data set illustrates how SCR may be used to condition for significant residual spatial structure and to identify additional predictors associated with residual spatial structure. Finally, I argue that all variance is spatially structured, hence spatial independence is best characterized by a lack of excess variance at any spatial scale, i.e., spatial white noise.
\end{abstract}

Key words: linear regression; Moran's eigenvector maps; multivariate ordination; oribatid mite species data set; spatial analysis; spatial autocorrelation; spatial filtering; spatial structure of ecological data; statistical hypothesis testing

\section{INTRODUCTION}

Linear regression is the workhorse of statistical modeling in many disciplines, including such disparate fields as ecology, social sciences, or econometrics and finance. Linear regression models a univariate response $\mathbf{y}$ as a linear combination of a set of predictors $\mathbf{X}$ by fitting a vector $\mathbf{b}$ of regression coefficients (Fig. 1). Linear regression has been extended to generalized linear regression that can accommodate many different types of response variables, and to the general linear model, $\mathbf{Y} \sim \mathbf{B X}+\mathbf{U}$, which allows for multivariate response data $\mathbf{Y}$ with multivariate error $\mathbf{U}$ and unifies commonly used methods including $t$ test, ANOVA,

Manuscript received 30 October 2012; revised 19 February 2013; accepted 8 April 2013. Corresponding Editor: B. D. Inouye.

${ }^{1}$ E-mail: helene.wagner@utoronto.ca
ANCOVA, and redundancy analysis (RDA). Hence, our understanding of the linear regression model is fundamental to almost any area of quantitative research.

The basic assumption of independent errors (i.e., residuals) is typically violated in spatial data sets (e.g., maps or images), and in time series, where nearby observations often are more similar than distant ones. How to build spatial or temporal structure into the linear regression model to analyze series of nonindependent data has been a long-standing challenge to researchers in many fields (Dormann et al. 2007, Beale et al. 2010). Spatial filtering presents one approach, where the variation in the data is modeled by a set $\mathbf{V}$ of uncorrelated (orthogonal) component patterns that represent different spatial or temporal scales of variation (see below; Fig. 2). Such spatial filtering conceptualizes space as a structuring process and thus uses the component patterns as additional predictor variables 

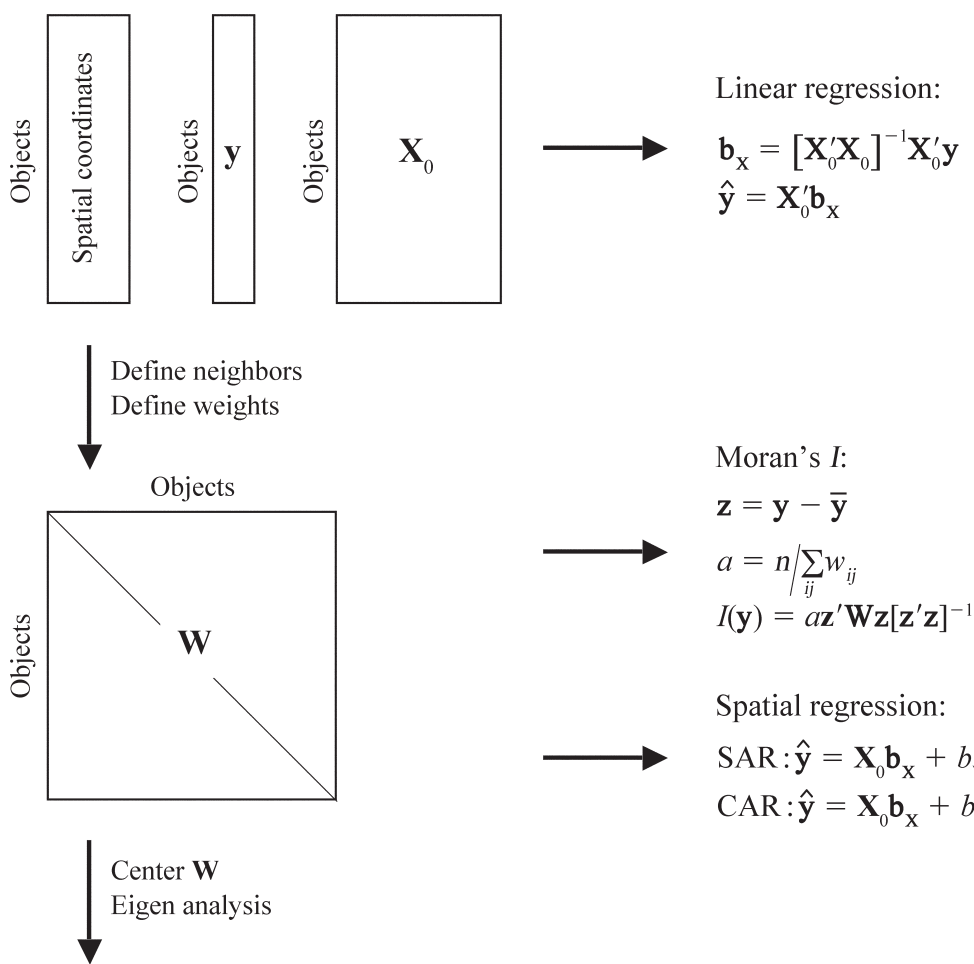

Linear regression:

$\longrightarrow \mathrm{b}_{\mathrm{X}}=\left[\mathrm{X}_{0}^{\prime} \mathrm{X}_{0}\right]^{-1} \mathbf{X}_{0}^{\prime} \mathbf{y}$

$\hat{\mathbf{y}}=\mathbf{X}_{0}^{\prime} \mathbf{b}_{\mathrm{x}}$

$=a \mathbf{z}^{\prime} \mathbf{W} \mathbf{z}\left[\mathbf{z}^{\prime} \mathbf{z}\right]^{-1}$

Spatial regression:

$\longrightarrow \quad$ SAR: $\hat{\mathbf{y}}=\mathbf{X}_{0} \mathbf{b}_{\mathbf{x}}+b_{\mathbf{w}} \mathbf{W}\left[\mathbf{y}-\mathbf{X}_{0} \mathbf{b}_{\mathbf{x}}\right]$

CAR: $\hat{\mathbf{y}}=\mathbf{X}_{0} \mathbf{b}_{\mathbf{x}}+b_{\mathbf{w}} \mathbf{W}[\mathbf{y}]$
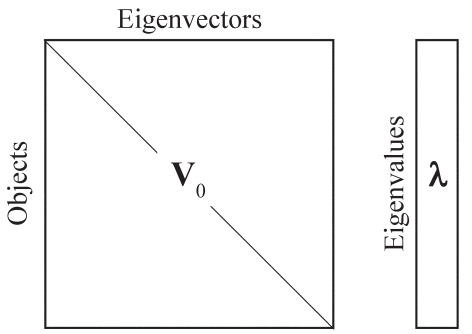

Moran's I:

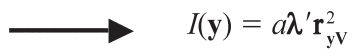

Select set of significant patterns $\mathbf{V}_{K}$ Include set $K$

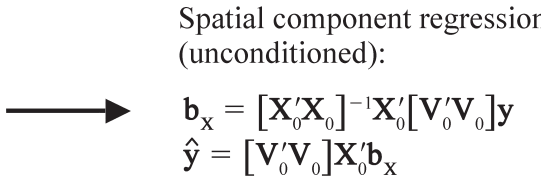

Select set of significant patterns $\mathbf{V}_{K}$ Exclude set $K$

Spatial filtering:

$\mathbf{b}_{\mathbf{x}}=\left[\mathbf{X}_{0}^{\prime} \mathbf{X}_{0}\right]^{-1} \mathbf{X}_{0}^{\prime} \mathbf{y}$

$\mathbf{b}_{K}=\left[\mathbf{V}_{K}^{\prime} \mathbf{V}_{K}\right]^{-1} \mathbf{V}_{K} \mathbf{y}$

$\hat{\mathbf{y}}=\mathbf{X}_{0} \mathbf{b}_{\mathbf{x}}+\mathbf{V}_{K} \mathbf{b}_{K}$

Spatial component regression (conditioned):

$$
\begin{aligned}
& \mathbf{b}_{\mathrm{XK}^{\mathrm{C}}}=\left[\mathbf{X}_{0}^{\prime} \mathbf{X}_{0}\right]^{-1} \mathbf{X}_{0}^{\prime}\left[\mathbf{V}_{0 K^{C}}{ }^{\prime} \mathbf{V}_{0 K^{C}}\right] \mathbf{y} \\
& \hat{\mathbf{y}}=\left[\mathbf{V}_{0 K^{C}} \mathbf{V}_{0 K^{\mathrm{C}}}\right] \mathbf{X}_{0} \mathbf{b}_{\mathrm{XK}^{\mathrm{C}}}
\end{aligned}
$$

FIG. 1. Flowchart of spatial component regression (SCR) and related methods (CAR, conditional autoregressive method; SAR, simultaneous autoregressive method). The symbol $\mathbf{y}$ refers to the univariate response, $\mathbf{X}_{0}$ to the matrix of predictors including a vector of 1's for the intercept, $\hat{\mathbf{y}}$ to the vector of fitted values, $\overline{\mathbf{y}}$ to the mean of $\mathbf{y}$, and $n$ to sample size. The vector $\mathbf{b}_{\mathbf{X}}$ is the vector of regression coefficients for the predictors in $\mathbf{X}_{0}, b_{\mathbf{W}}$ is the regression coefficient for the spatial term defined by spatial-weight matrix $\mathbf{W}$ (with elements $w_{i j}$ ), and $\mathbf{b}_{\mathbf{K}}$ is the vector of regression coefficients for the component patterns $k$ in set $K$ that are included as predictors in spatial filtering. $\mathbf{V}_{0 K^{c}}$ refers to the matrix of eigenvectors of $\mathbf{W}$, including any eigenvector $\mathbf{v}_{0}$ with zero variance, excluding those (significant) component patterns $\mathbf{v}_{k}$ that are included in set $K$, and $\lambda$ denotes the vector of eigenvalues of $\mathbf{W}$. $I(\mathrm{y})$ denotes global Moran's $I$ of variable $y$. Finally, $\mathbf{r}_{\mathbf{y v}}^{2}$ refers to the vector of squared correlations between response $\mathbf{y}$ and the component patterns (spatial eigenvectors) in $\mathbf{V}$, excluding any eigenvector $\mathbf{v}_{0}$ with zero variance. For additional explanation of symbols; see Introduction. 
to account for spatial or temporal structure in the data (Diniz-Filho et al. 1998, Borcard and Legendre 2002, Griffith 2000, Dray et al. 2006, Griffith and Peres-Neto 2006, Patuelli et al. 2011, Legendre and Legendre 2012). Here, I argue that space is not an ecologically meaningful predictor (Hawkins and Diniz-Filho 2004) but a dimension of the data, and that statistical methods used for selection of spatial predictors may not appropriately reflect the statistical properties of component patterns. I propose a new solution of "spatial component regression" (SCR), where the component patterns are used to partition the correlation between response and predictors into scale-dependent components. The proposed partitioning allows the prediction of spatial or temporal structure of fitted and residual values, and it provides an alternative to spatial filtering that takes into account the statistical properties of component patterns. Beyond definition of neighbors and their respective weights, the method does not require assumptions on the nature of the underlying patterns, as the decomposition into component patterns preserves $100 \%$ of the variation of the original variables. This novel integration of methods leads to some interesting insights and challenges us to re-think spatial analysis, spatial filtering, multivariate ordination, and the linear regression model. All analyses were performed in $\mathrm{R}$ version 2.15.2 (R Development Core Team 2012) using libraries "spdep" (Bivand et al. 2012) and "vegan" (Oksanen et al. 2012), and $\mathrm{R}$ code to reproduce the empirical examples can be found in Supplements 1 and 2.

\section{Spatial autocorrelation}

Spatial autocorrelation is commonly considered either a nuisance or a property of interest (Legendre 1993). The nuisance arises from the fact that spatial autocorrelation in the residuals violates the assumption of independent errors in a linear regression model and may affect parameter estimates (Lennon 2000, Diniz-Filho et al. 2003, Dormann et al. 2007). Spatial structure in the residuals may result from a spatial process such as dispersal (autocorrelation in the strict sense; Legendre 1993), or it may reflect the omission of an important spatially structured predictor and thus model misspecification. In contrast, in a correctly specified model, spatial dependence (sensu Legendre 1993) induced by a spatially independent response to a spatially structured environmental factor should not result in spatial autocorrelation of the residuals.

Moran's $I$ is the most widely used statistic to estimate spatial autocorrelation. It mostly varies between -1 and +1 (slight deviations may occur for small samples; de Jong et al. 1984), with an expected value of $\mathrm{E}[I]=-1 /(n$ - 1) for a sample of $n$ spatially independent observations (Moran 1950). Global Moran's I quantifies the covariance among neighboring values as compared to the covariance among all observed values of the same variable. Positive values indicate positive spatial auto-
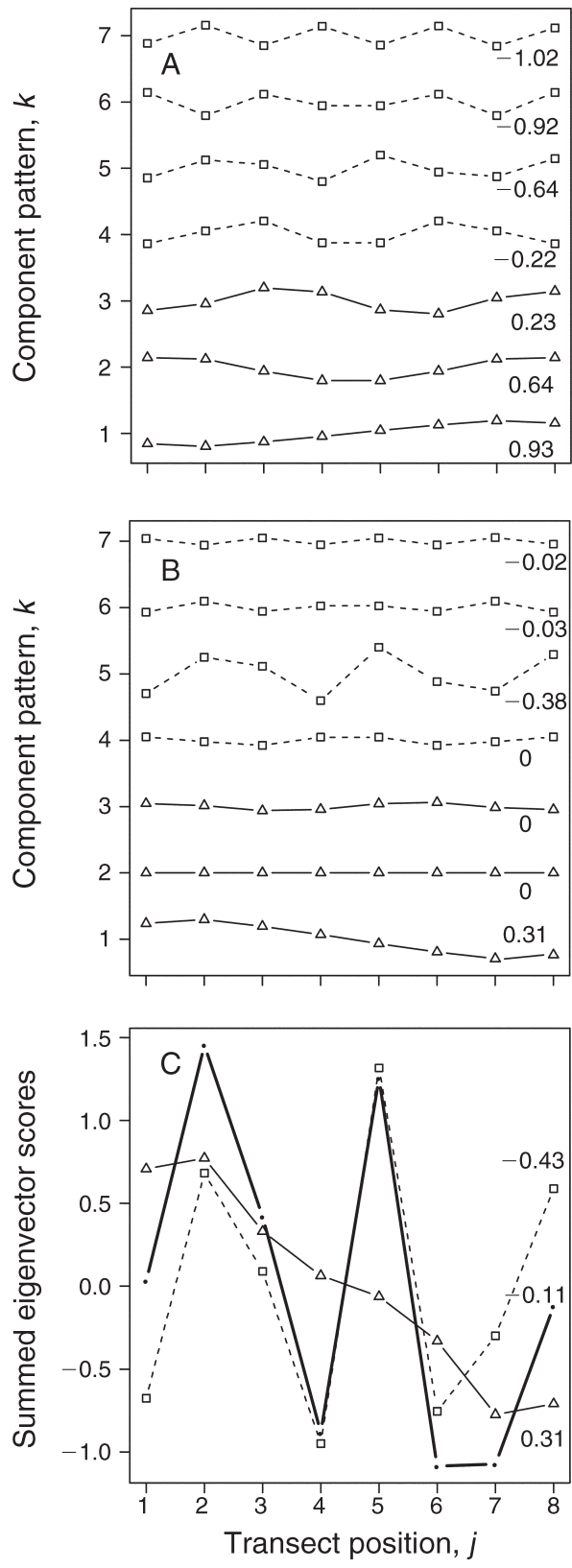

FIG. 2. (A) Variation of component patterns $k$ based on the sampling locations alone and (B) corresponding spatial patterns in the standardized response $\mathbf{y}$ along a transect of $n=8$ sampling locations. Solid lines with open triangle symbols indicate component patterns with positive eigenvalues, dotted lines with open squares are those with negative eigenvalues, and numbers on the right side of each plot indicate Moran's $I$ associated with each pattern. (C) Illustration of how the variation of the values of $\mathbf{y}$ along the transect (bold solid line with solid circles), and corresponding Moran's I, can be decomposed into the sum over component patterns (as expressed in $\mathbf{y}$ ) with positive spatial autocorrelation (thin solid line with open triangles), and the sum over component patterns with negative spatial autocorrelation (dotted line with open squares). 
correlation, where nearby observations are more similar on average than distant ones, whereas negative values indicate negative spatial autocorrelation, where nearby observations are more dissimilar than distant ones. The calculation of Moran's I involves definition of a spatial weight matrix $\mathbf{W}$, which assigns a weight $w_{i j}$ to each neighbor $j$ of sampling unit $i$, with $w_{i j}=0$ for nonneighbors (Fig. 1). Neighbor weights $w_{i j}$ can be defined in many ways, including binary weights ( 1 if neighbor, 0 otherwise) or weights proportional to a function of geographic distance between neighbors (Dray et al. 2006, Fortin et al. 2012, Legendre and Legendre 2012). In matrix notation, Moran's $I(\mathrm{y})$ for response $\mathbf{y}$ is then calculated as

$$
I(\mathbf{y})=a \mathbf{z}^{\prime} \mathbf{W} \mathbf{z}\left[\mathbf{z}^{\prime} \mathbf{z}\right]^{-1}
$$

where $a=n / \Sigma_{i j} w_{i j}$ is a constant and $\mathbf{z}$ is obtained by centering $\mathbf{y}$ (i.e., removing the mean). Matrix $\mathbf{W}$ is also used in spatial regression (Anselin 2002) to model autocorrelation among neighboring observations (Fig. 1) as a neighborhood function of either the residuals (simultaneous autoregressive error model, SAR; Whittle 1954) or the response (conditional autoregressive model, CAR; Besag 1974).

\section{Spatial filtering with Moran's eigenvector map analysis}

This paper is motivated by the notion that currently used methods of spatial filtering (Dray et al. 2006, Griffith and Peres-Neto 2006) go in the right direction but do not provide the final answer to the challenge of appropriately representing spatial or temporal patterns in the linear regression model. This section summarizes the currently recommended procedure of spatial filtering based on Moran's eigenvector map analysis (MEM; Dray et al. 2006, Legendre and Legendre 2012).

Spatial filtering starts with the definition of neighbors and weights, i.e., the construction of spatial weight matrix $\mathbf{W}$ (see above). MEM requires $\mathbf{W}$ to be symmetric (for an extension to asymmetric weights, see Blanchet et al. 2008b), which is achieved by calculating $\mathbf{W}_{\text {sym }}=(\mathbf{W}+$ $\left.\mathbf{W}^{\prime}\right) / 2$. Finally, the matrix $\mathbf{W}_{\text {sym }}$ is rescaled by removing row and column means prior to eigen-analysis to ensure that eigenvectors are uncorrelated and correctly scaled (see R code in Supplement 1).

Eigen-analysis of the rescaled spatial weight matrix derived from $n$ sampling locations results in an $n \times n$ matrix $\mathbf{V}_{0}$ of eigenvectors $\mathbf{v}_{k}$ (i.e., component patterns $k)$. However, one eigenvector $\mathbf{v}_{0}$ typically has zero variance (all its values are $1 / \mathcal{V}(n-1))$ and an associated eigenvalue of 0 (or nearly zero due to computational constraints), and $\mathbf{v}_{0}$ is typically discarded to avoid computational problems. I will use $\mathbf{V}_{0}$ to refer to the square matrix with all $n$ eigenvectors (needed below), and $\mathbf{V}$ for the $n \times(n-1)$ matrix, where eigenvector $\mathbf{v}_{0}$ was dropped. The variance in any response $\mathbf{y}$ can be fully modeled by the spatial patterns represented by $\mathbf{V}$, which were derived from the sampling locations alone. The vector of the squared correlations $\mathbf{r}_{\mathbf{y V}}^{2}$ between response $\mathbf{y}$ and each eigenvector in $\mathbf{V}$ provides a scalogram $\mathbf{s}$ (Legendre and Legendre 2012) that summarizes how the variance of $\mathbf{y}$ is distributed across scales.

The interpretation of $\mathbf{V}$ capitalizes on the property that the eigenvalue $\lambda_{k}$ of a component pattern $k$ is proportional to its Moran's $I\left(\mathbf{v}_{k}\right)$ (Fig. 1; Griffith 2000, Dray et al. 2006). The component patterns are thus sorted by the scale of pattern that they represent, from largest to smallest. Positive eigenvalues $\lambda_{k}$ represent component patterns $k$ with positive spatial autocorrelation (global structures), whereas negative eigenvalues represent patterns with negative autocorrelation (local structures; Dray 2011). As an alternative to Eq. 1, Moran's $I(\mathbf{y})$ for a variable $\mathbf{y}$ can be calculated from the product of the eigenvalue $\lambda_{k}$ and the correlation $r_{\mathbf{y} \mathbf{V}}$ between $\mathbf{y}$ and the component patterns in matrix $\mathbf{V}$ (Fig. 1; Dray 2011). In matrix notation:

$$
I(\mathbf{y})=a \lambda^{\prime} \mathbf{r}_{\mathbf{y V}}^{2}
$$

Spatial filtering uses a subset $K$ of component patterns as regression predictors to model spatial autocorrelation in the data (Fig. 1). To avoid a large number of additional predictors, different methods for selecting significant component patterns $k$ have been proposed (Diniz-Filho and Bini 2005, Tiefelsdorf and Daniel 2007, Blanchet et al. 2008a, Jombart et al. 2009, Diniz-Filho et al. 2012a): (1) selection may be based on the association between $\mathbf{V}$ and $\mathbf{y}$ or $\mathbf{V}$ and the residuals of $\mathbf{y}$, (2) selection may be based on the variance $\mathbf{r}_{\mathbf{y V}}^{2}$ or Moran's $I_{k}(\mathbf{y})$ associated with component patterns, and (3) component patterns may be tested sequentially (from largest-scale to smallest-scale patterns) or independently. Finally, matrix $\mathbf{V}_{K}$, which contains only the selected subset $K$, is added to the linear regression model as a set of additional predictors (Fig. 1).

Using component patterns as predictors has statistical problems that have received little attention to date. First, the $n-1$ eigenvectors in $\mathbf{V}$ together explain $100 \%$ of the variance in $\mathbf{y}$ (Rohlf 2001). The expected variance of a normally distributed variable explained by a single eigenvector is thus $1 /(n-1)$, and not zero. Hence, testing against a null hypothesis of zero variance explained is not appropriate for the selection of significant component patterns. Second, using $t$ tests for the regression slope of component patterns is not appropriate because the slopes are not independent. Third, testing Moran's $I_{k}(\mathbf{y})$ associated with component pattern $k$ against the null hypothesis of $\mathrm{E}\left[I_{k}(\mathbf{y})\right]=-1 /(n-1)$, i.e., the expected global Moran's $I$, is not appropriate as the expected value depends on $k: \mathrm{E}\left[I_{k}(\mathbf{y})\right]=I\left(\mathbf{v}_{k}\right) \times(1 /(n-1))$. Recently, more appropriate tests have been proposed (Jombart et al. 2008, Diniz-Filho et al. 2012b).

Excess variance at any particular scale may indicate model misspecification, where an important process was not accounted for (Anselin 2002, Borcard et al. 2004, Wagner and Fortin 2005). If processes may occur at any scale and each process has its characteristic spatial scale (Wiens 1989), excess variance at any scale may indicate 
model misspecification. Hence, spatial filtering should not be restricted to component patterns with large Moran's I (e.g., Griffith 2000).

Nevertheless, the spatial scale of a missing factor will determine how the spatial structure in the residuals affects significance tests in regression analysis. Strong positive spatial autocorrelation is likely to inflate type 1 error by reducing effective sample size (Griffith 2005), whereas strong negative spatial autocorrelation may have the opposite effect (Griffith and Arbia 2010), and significant component patterns with near-zero eigenvalues may have little or no effect on significance tests for predictors $\mathbf{X}$.

\section{Summary of ordinary least-squares regression}

Before we can expand the linear regression model to incorporate spatial or temporal structure in the data, we need to define the relevant properties of the basic linear regression model as a reference. It is assumed that the reader has basic familiarity with ordinary least-squares regression, where a response variable $\mathbf{y}$ is predicted from a matrix of predictors $\mathbf{X}$ by the following formula (in matrix notation):

$$
\mathbf{y}_{\text {fit }}=\mathbf{X}_{0}\left(\mathbf{X}_{0}^{\prime} \mathbf{X}_{0}\right)^{-1} \mathbf{X}_{0}^{\prime} \mathbf{y} .
$$

Note that all predictors in $\mathbf{X}$ should be centered (i.e., zero means) before using Eq. 3. Unless the response $\mathbf{y}$ is centered as well, $\mathbf{X}$ should include a vector of 1's for the intercept $b_{0}$. We will use $\mathbf{X}_{0}$ for the matrix $\mathbf{X}$ that includes a column for the intercept, and $\mathbf{X}$ for the predictor matrix without such a term.

In multiple linear regression (i.e., with multiple predictors), multiple $R^{2}$ can be derived by

$$
R^{2}=\mathbf{r}_{\mathbf{y X}}^{\prime} \mathbf{R}_{\mathbf{X X}}^{-1} \mathbf{r}_{\mathbf{y X}}=\boldsymbol{\rho}_{\mathbf{y X}}^{\prime} \mathbf{r}_{\mathbf{y X}}
$$

where $\mathbf{r}_{\mathbf{y X}}$ is the vector of simple correlation coefficients between response $\mathbf{y}$ and each vector in predictor matrix $\mathbf{X}$ (without the intercept term to avoid problems of zero variance), and $\mathbf{R}_{\mathbf{X X}}$ is the correlation matrix of $\mathbf{X}$. Note that $\mathbf{r}_{\mathbf{y X}}{ }^{\prime} \mathbf{R}_{\mathbf{X X}}{ }^{-1}$ provides the vector $\boldsymbol{\rho}_{\mathbf{y X}}$ of partial correlation coefficients, which correspond to the standardized multiple regression coefficients (also known as beta coefficients).

\section{Basic Definition of Spatial Component Regression, SCR}

We can use matrix $\mathbf{V}$ derived from Moran's eigenvector map (MEM) analysis (previously described) to decompose the linear regression model into components associated with all or a subset $K$ of component patterns in $\mathbf{V}$.

Vector $\mathbf{r}_{\mathbf{y} \mathbf{V}}$ describes the linear correlation between response $\mathbf{y}$ and each component pattern $k$ in $\mathbf{V}$, and the scalogram $\mathbf{s}=\mathbf{r}_{\mathbf{y V}}^{2}$ sums to 1 . Similarly, matrix $\mathbf{R}_{\mathbf{X V}}$ contains the linear correlations between the predictor variables in $\mathbf{X}$ and matrix $\mathbf{V}$. The pairwise correlations between predictors in $\mathbf{X}$ and response $\mathbf{y}$ can be obtained from their correlations with $\mathbf{V}$ :

$$
\mathbf{r}_{\mathbf{y X}}=\mathbf{r}_{\mathbf{y V}}^{\prime} \mathbf{R}_{\mathbf{X V}} .
$$

By extension, we get $\mathbf{R}_{\mathbf{X X}}=\mathbf{R}_{\mathbf{X V}}{ }^{\prime} \mathbf{R}_{\mathbf{X V}}$.

We can reconstruct $\mathbf{y}$ completely as a linear function of $\mathbf{V}_{0}$. Note that we should use $\mathbf{V}_{0}$, with all $n$ eigenvectors, unless $\mathbf{y}$ is centered. This allows us to select a subset $K$ of component patterns $k$ in $\mathbf{V}_{0}$ :

$$
\mathbf{y}_{K}=\mathbf{V}_{0 K} \mathbf{V}_{0 K}^{\prime} \mathbf{y} \text {. }
$$

By extension, we get $\mathbf{X}_{K}=\mathbf{V}_{0 K} \mathbf{V}_{0 K}$ ' $\mathbf{X}$. We can thus predict $\mathbf{y}$ from $\mathbf{X}$ across a restricted range of spatial scales (conditioned SCR), excluding a subset $K$ (Fig. 1):

$$
\mathbf{y}_{\text {fit. } K^{\mathrm{c}}}=\mathbf{V}_{0 K^{\mathrm{c}}} \mathbf{V}^{\prime}{ }_{0 K^{\mathrm{c}}} \mathbf{X}_{0}\left(\mathbf{X}_{0}^{\prime} \mathbf{X}_{0}\right)^{-1} \mathbf{X}_{0}^{\prime} \mathbf{V}_{0 K^{\mathrm{c}}} \mathbf{V}^{\prime}{ }_{0 K^{\mathrm{c}}} \mathbf{y}
$$

where $\mathbf{V}_{0 K}{ }^{c}$ refers to matrix $\mathbf{V}_{0}$ without subset $K$ (i.e., the complement of $\left.K, K^{\mathrm{c}}\right)$. Each component pattern $k$ corresponds to one degree of freedom in the residuals, so that the residual degrees of freedom should be adjusted by the number of component patterns in subset $K$ that was excluded. If all component patterns in $\mathbf{V}_{0}$ are retained (i.e., $K=\{\}$ ), Eq. 7 will result in unconditioned SCR (Fig. 1).

The vector $\boldsymbol{\rho}_{\mathbf{y} \mathbf{X}}{ }^{c}$ of partial regression coefficients of predictors $\mathbf{X}$, accounting for all other predictors in $\mathbf{X}$ and for component patterns in set $K$, can be found as:

$$
\boldsymbol{\rho}_{\mathbf{y X} K^{\mathrm{c}}}=\mathbf{r}_{\mathbf{y V} K^{\mathrm{c}}}^{\prime} \mathbf{R}_{\mathbf{X V} K^{\mathrm{c}}}\left(\mathbf{R}_{\mathbf{X V} K^{\mathrm{c}}}^{\prime} \mathbf{R}_{\mathbf{X V} K^{\mathrm{c}}}\right)^{-1} .
$$

The proportion of variance explained, $R^{2}$, can be partitioned into two components:

$R^{2}=R_{\mathbf{V} K}^{2}+R_{\mathbf{X} K^{\mathrm{c}}}^{2}=\mathbf{r}_{\mathbf{y V} K}^{\prime} \mathbf{r}_{\mathbf{y V} K}+\boldsymbol{\rho}_{\mathbf{y X X}} K^{\mathbf{c}} \mathbf{R}_{\mathbf{X V} K^{\mathrm{c}}}^{\prime} \mathbf{r}_{\mathbf{y V} K^{\mathrm{c}}}$.

Borrowing terminology from redundancy analysis (RDA; Legendre and Legendre 2012), $R^{2}{ }_{\mathbf{V} K}$ is the conditioned variance, i.e., the proportion of the variance of $\mathbf{y}$ explained by the set of eigenvectors $\mathbf{V}_{K}$, and $R^{2}{ }_{\mathbf{X}}{ }^{\mathrm{c}}$ is the constrained variance, i.e., the proportion of the variance of $\mathbf{y}$ explained by predictors $\mathbf{X}$ as conditioned by $\mathbf{V}_{K}$.

\section{Identification of significant spatial structures in the residuals}

A main goal of building space into the linear regression model is to remove spatial autocorrelation from the residuals. The correlation between the residuals of an initial model and the eigenvectors in $\mathbf{V}$ can thus be used to identify the subset $K$ of component patterns that should be accounted for in the analysis (Bini et al. 2009). Here I show how we can predict this correlation, for the fitted ( $\left.\mathbf{r}_{\mathbf{y} \text {.fit.v }}\right)$ and residual $\left(\mathbf{r}_{\mathbf{y} . \text { res. }} \mathbf{v}\right)$ values, in the framework of SCR.

In simple regression with a single predictor $\mathbf{x}$, the fitted values $\mathbf{y}_{\text {fit }}$ will completely inherit the spatial structure from $\mathbf{x}$, so that $\mathbf{r}_{\mathbf{y} \text {.fit. } \mathbf{v}}=\mathbf{r}_{\mathbf{x} \mathbf{v}}$. In multiple regression, the spatial structure $\mathbf{r}_{\mathbf{y} \text {.fit.v }}$ of $\mathbf{y}_{\text {fit }}$ is a weighted mean of the spatial structure $\mathbf{R}_{\mathbf{X V}}$ of the predictors, 
TABLE 1. Values of a single standardized response variable $\mathbf{y}$ (e.g., the abundance of a single species) simulated at $n=8$ sampling locations $j$ along a regular transect, and decomposition of $\mathbf{y}$ into additive components $\mathbf{y}_{j k}$ proportional to each component pattern $k$, where $k=\{1,2, \ldots, n-$ $1\}$.

\begin{tabular}{|c|c|c|c|c|c|c|c|c|}
\hline \multirow{2}{*}{$\begin{array}{l}\text { Sampling } \\
\text { location, } j\end{array}$} & \multirow[b]{2}{*}{ Response, $\mathbf{y}$} & \multicolumn{7}{|c|}{ Additive components of response variable } \\
\hline & & $\mathbf{y}_{j 1}$ & $\mathbf{y}_{j 2}$ & $\mathbf{y}_{j 3}$ & $\mathbf{y}_{j 4}$ & $\mathbf{y}_{j 5}$ & $\mathbf{y}_{j 6}$ & $\mathbf{y}_{j 7}$ \\
\hline 1 & 0.03 & 0.59 & -0.0007 & 0.11 & 0.13 & -0.74 & -0.17 & 0.10 \\
\hline 2 & 1.46 & 0.74 & -0.0006 & 0.04 & -0.05 & 0.63 & 0.24 & -0.14 \\
\hline 3 & 0.42 & 0.49 & 0.0003 & -0.16 & -0.19 & 0.29 & -0.14 & 0.13 \\
\hline 4 & -0.89 & 0.17 & 0.0009 & -0.11 & 0.12 & -1.01 & 0.07 & -0.13 \\
\hline 5 & 1.26 & -0.17 & 0.0009 & 0.11 & 0.12 & 1.01 & 0.07 & 0.13 \\
\hline 6 & -1.09 & -0.49 & 0.0003 & 0.16 & -0.19 & -0.29 & -0.14 & -0.13 \\
\hline 7 & -1.07 & -0.74 & -0.0006 & -0.04 & -0.05 & -0.63 & 0.24 & 0.14 \\
\hline 8 & -0.12 & -0.59 & -0.0007 & -0.11 & 0.13 & 0.74 & -0.17 & -0.10 \\
\hline
\end{tabular}

weighted by the partial beta coefficients and scaled by the square root of the explained variance $R^{2}$ :

$$
\begin{aligned}
\mathbf{r}_{\mathbf{y} . \text { fit. }} & =\left(R^{2}\right)^{-0.5} \mathbf{R}_{\mathbf{X V}} \boldsymbol{\rho}_{\mathbf{y X}} \\
& =\left(\mathbf{r}_{\mathbf{y V}}^{\prime} \mathbf{R}_{\mathbf{X V}} \boldsymbol{\rho}_{\mathbf{y X}}\right)^{-0.5} \mathbf{r}_{\mathbf{y V}}^{\prime} \mathbf{R}_{\mathbf{X V}}\left(\mathbf{R}_{\mathbf{X V}}^{\prime} \mathbf{R}_{\mathbf{X V}}\right)^{-1} \mathbf{R}_{\mathbf{X V}} .
\end{aligned}
$$

The spatial structure of the residuals $\left(\mathbf{y}-\mathbf{y}_{\text {fit.K }}\right)$ derives from the difference between the spatial structure of the response $\mathbf{y}$ and its fitted values, scaled by the square-root of the residual variance of $\mathbf{y}$ :

$$
\mathbf{r}_{\mathbf{y . r e s .}}=\left(1-\mathbf{r}_{\mathbf{y V}}^{\prime} \mathbf{R}_{\mathbf{X V}} \boldsymbol{\rho}_{\mathbf{y X}}\right)^{-0.5}\left(\mathbf{r}_{\mathbf{y V}}-\mathbf{R}_{\mathbf{X V}} \boldsymbol{\rho}_{\mathbf{y X}}\right) \text {. }
$$

Note that only $\mathbf{r}_{\mathbf{y V}}, \mathbf{R}_{\mathbf{X V}}$, and $n$ are needed to predict residual spatial structure $\mathbf{r}_{\text {y.res.v. }}$.

We can compare the predicted variance of the residuals associated with each component pattern $k$,

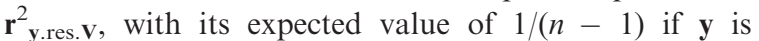
associated equally with all component patterns. Jombart et al. (2008) demonstrated the validity of a permutation test, where the values of the residuals $\mathbf{y}$ are permuted to obtain, for each permutation, the maximum of $\mathbf{r}^{2} \mathbf{y . r e s . v}$. Observed values in $\mathbf{r}_{\mathbf{y} \text {.res. } \mathbf{v}}$ larger than $95 \%$ of the maxima obtained by permutation indicate significant component patterns. To control for significant residual spatial structure by spatial filtering (i.e., conditioning), we can thus define subset $K$ in Eqs. 7-9 as the set of

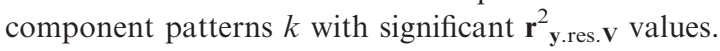

\section{Illustration of component patterns with simulated data set}

This section illustrates the spatial decomposition into component patterns for a univariate response $\mathbf{y}$, such as the abundance of a single species simulated along a regular transect of $n=8$ sampling locations (Table 1). See Supplement 1 for $\mathrm{R}$ code used to analyze the simulated data set and implement calculations for spatial component regression (SCR).

Fig. 2A shows all seven component patterns $k$ with nonzero eigenvalues, derived (using Moran's eigenvector map, MEM) from the $n=8$ sampling locations, where adjacent quadrats were considered neighbors $j$, and weights $w_{\mathrm{ij}}$ were defined to sum to 1 for each sampling location $i$. The response $\mathbf{y}$ that was simulated at the $n=8$ sampling locations can be decomposed by the component patterns using Eq. 6 (Fig. 2B). Rescaling the component patterns (Fig. 2A) by their correlation with $\mathbf{y}$ shows to what degree each component pattern is expressed in the simulated response $\mathbf{y}$ (Fig. 2B).

Summing the patterns in Fig. 2B over all component patterns $k$ recreates the original values of $\mathbf{y}$ (Fig. 2C, bold solid line). Alternatively, summing over component patterns with positive spatial autocorrelation (solid line in Fig. 2B) returns the variation in $\mathbf{y}$ associated with global structures, i.e., the variation in local mean (Fig. $2 \mathrm{C}$, thin solid line). Conversely, summing over component patterns with negative spatial autocorrelation (dashed lines in Fig. 2B) returns the variation in $\mathbf{y}$ associated with local structures, i.e., the variation around the local mean (Fig. 2C, dashed line). Adding the two components results in the original values of $\mathbf{y}$. All Moran's I components associated with global and local structures sum to $I(\mathbf{y})=0.313-0.428=-0.115$ (Fig. 2C).

Most of the variance in response $\mathbf{y}$ was associated with component patterns $k=1$ and $k=5$ (Fig. 3A). In contrast, a variable that is perfectly spatially independent would be expected to have equal variance associated with each component pattern, i.e., exhibit spatial white noise (Lennon 2000; Fig. 3B). A perfect linear trend (here: transect position $j$ ) will mostly be associated with the largest-scale pattern $k=1$, but show some variance associated with other component patterns as well, including some fine-scale patterns (Fig. 3C).

\section{Extension to multivariate response data}

Ordination methods are frequently used in community ecology to reduce the information contained in a potentially large number of species variables so as to extract the structure shared by many species (indirect ordination such as principal-component analysis PCA, based on species data only) or to relate multispecies structures to explanatory variables that represent ecological gradients (direct ordination such as redundancy analysis RDA). Redundancy analysis consists of 
two steps: multiple regression of each species variable in $\mathbf{Y}$ on the predictors $\mathbf{X}$, and subsequent PCA, separately for the fitted and the residual values (Legendre and Legendre 2012).

Assume that instead of a univariate response $\mathbf{y}$, we have an $n \times m$ matrix $\mathbf{Y}$ with abundances of $m$ species (columns) observed at $n$ sampling locations (rows). Vector $\mathbf{r}_{\mathbf{y} \mathbf{V}}$ thus needs to be replaced by a matrix $\mathbf{R}_{\mathbf{Y V}}$, and Eqs. 8 and 9 are replaced by

$$
\mathbf{P}_{\mathbf{Y X} K^{\mathrm{c}}}=\mathbf{R}_{\mathbf{Y V} K^{\mathrm{c}}}^{\prime} \mathbf{R}_{\mathbf{X V} K^{\mathrm{c}}}\left(\mathbf{R}_{\mathbf{X V} K^{\mathrm{c}}}^{\prime} \mathbf{R}_{\mathbf{X V} K^{\mathrm{c}}}\right)^{-1}
$$

and

$$
\begin{aligned}
R^{2} & =R_{\mathbf{Y V} K}^{2}+R_{\mathbf{X} K^{\mathrm{c}}}^{2} \\
& =\operatorname{tr}\left(\mathbf{C R}^{\prime}{ }_{\mathbf{Y V} K} \mathbf{r}_{\mathbf{y} \mathbf{V} K}\right)+\operatorname{tr}\left(\mathbf{C} \mathbf{P}_{\mathbf{Y} \mathbf{X} K^{\mathrm{c}}} \mathbf{R}^{\prime}{ }_{\mathbf{X V} K^{\mathrm{c}}} \mathbf{R}_{\mathbf{Y V} K^{\mathrm{c}}}\right)
\end{aligned}
$$

where the trace $\operatorname{tr}()$ denotes the sum of the diagonal elements of a square matrix. $\mathbf{C}$ is an $m \times m$ matrix whose diagonal elements specify column (species) weights (with $\operatorname{tr}(\mathbf{C})=1$ ) and all off-diagonal elements are 0 . Column weights may be defined, e.g., with equal weights for all species or with weights proportional to the variance of each species variable. Note that it may often be advisable to apply Hellinger transformation to $\mathbf{Y}$ before performing multivariate regression of species data (Legendre and Gallagher 2001); this transformation rescales values so that the squared new values sum to 1 for each sampling location (row).

Multivariate Moran's $I(\mathbf{Y})$ is obtained as a weighted mean of Moran's I of the species variables, weighted by column weights in C. In matrix notation,

$$
I(\mathbf{Y})=\lambda^{\prime} \mathbf{s}=\lambda^{\prime} \mathbf{R}_{\mathbf{Y V}}^{2} \mathbf{C 1}
$$

where $\mathbf{1}$ is an $n \times 1$ vector of values 1 . Note that $\lambda^{\prime} \mathbf{R}^{2} \mathbf{Y V}$ provides the values of Moran's $I$ for each species, and $\mathbf{R}_{\mathbf{Y V}}^{2} \mathbf{C 1}$ provides the multivariate scalogram $\mathbf{s}$.

To identify set $K$ of significant component patterns based on the multivariate scalogram $\mathbf{s}$ of the residuals, the permutation test described above is modified by permuting rows of the matrix of residuals $\mathbf{Y}_{\text {res }}$ and determining for each permutation the maximum of $\mathbf{s}$ (Jombart et al. 2008).

\section{Illustration with multivariate oribatid mite data set}

The well-known mite data set (Borcard et al. 1992, Borcard and Legendre 1994, Griffith and Peres-Neto 2006, Jombart et al. 2009) contains abundance data of 35 oribatid mite species observed at 70 sampling locations irregularly spaced within a study area of 2.6 $\times 10 \mathrm{~m}$ (data available in R libraries "vegan" or "ade4"). The following explanatory variables were measured at each sampling location: substrate density, water content (both quantitative), microtopography (binary), shrub density (ordinal, three levels), and substrate type (nominal, seven levels). See Supplement 2 for details of the analysis below.

The original values Y (Table 2: Model 1), which represent Hellinger-transformed species abundances,
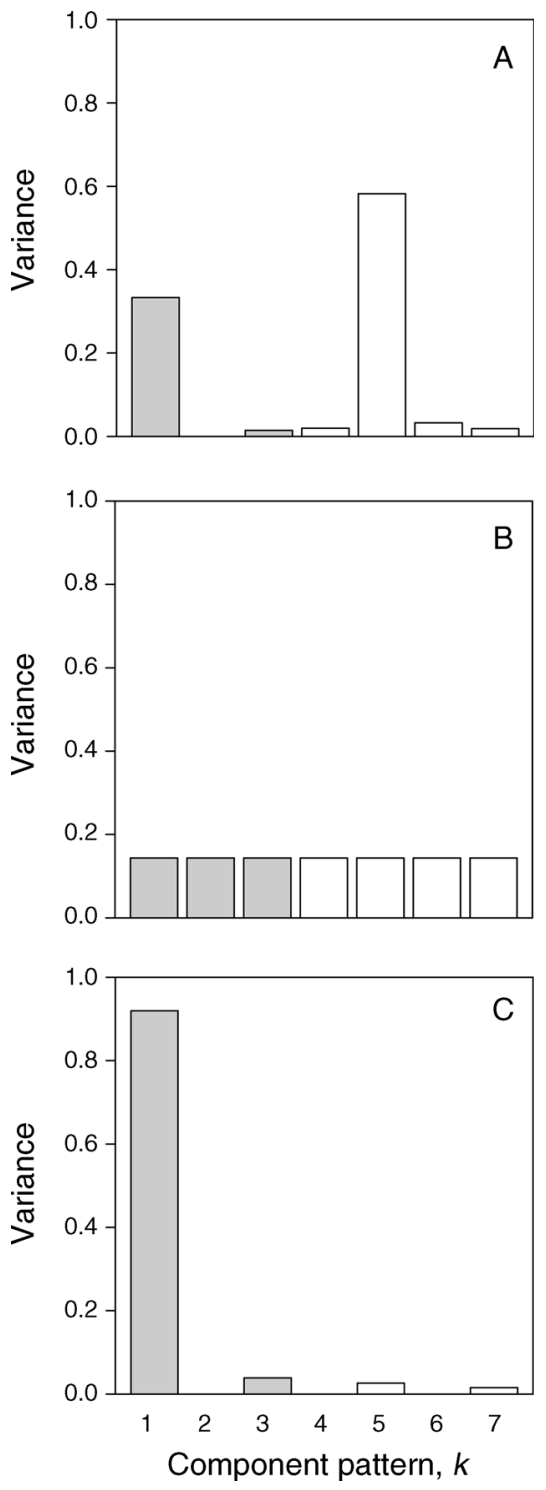

FIG. 3. Each barplot shows the variance associated with component patterns $k$ (A) for the simulated response $\mathbf{y}$, (B) for an ideally distributed random variable with equal variance associated with each component pattern, and (C) for linear trend as in predictor $\mathbf{x}_{1}$. Only component patterns with nonzero eigenvector and nonzero variance are shown. Gray bars indicate component patterns with positive spatial autocorrelation (i.e., $\lambda_{k}>0$ ), and white bars indicate those with negative spatial autocorrelation $\left(\lambda_{k}<0\right)$.

showed a single significant component pattern with $K$ $=\{1\}$. A total of 22 species showed significant patterns that were all associated with the first five component patterns, thus representing global structures.

To illustrate the application of SCR (spatial component regression), I fitted an initial multivariate regression model using only the two quantitative variables as predictors (Table 2: Model 2). Substrate density and water content partly explained the spatial structure in $\mathbf{Y}$, though there remained significant positive spatial 
TABLE 2. Variance components and significant component patterns identified for the oribatid mite data set, based on four different models.

\begin{tabular}{|c|c|c|c|c|c|c|c|c|c|}
\hline \multicolumn{3}{|c|}{ Model } & \multicolumn{3}{|c|}{ Variance components: } & \multicolumn{3}{|c|}{ Significant component patterns§ } & \multirow[b]{2}{*}{$\begin{array}{c}\text { Moran's } \\
I \Phi \\
\end{array}$} \\
\hline No. & Type† & $\begin{array}{l}\text { No. } \\
\text { predictors }\end{array}$ & Conditioned & Constrained & $\begin{array}{l}\text { Un- } \\
\text { constrained }\end{array}$ & $K_{\text {comm }}$ & $K_{\text {spp }}$ & $\begin{array}{l}\text { No. } \\
\text { species }\end{array}$ & \\
\hline 1 & intercept only & & & & 1.00 & 1 & $1,2,3,5$ & 22 & 0.42 \\
\hline 2 & unconditioned SCR & 2 & & 0.327 & 0.673 & 2,4 & $2,4,6,21$ & 8 & 0.19 \\
\hline 3 & $\begin{array}{l}\text { conditioned SCR with } \\
\qquad K=\{2,4\}\end{array}$ & 2 & 0.073 & 0.354 & 0.573 & & $3,6,21$ & 3 & 0.06 \\
\hline 4 & unconditioned SCR & 6 & & 0.460 & 0.540 & & 21,57 & 2 & 0.04 \\
\hline
\end{tabular}

Note: Blank cells mean those parameters do not exist.

$\dagger$ Model 1 is an intercept-only model; Model 2 is an unconditioned SCR (spatial component regression) with two predictors, "Water content" and "Substrate density"; Model 3 is a conditioned SCR with the same two predictors but conditioned for two component patterns; and Model 4 is an unconditioned SCR with four additional dummy variables representing microtopography, shrub density (two dummy variables), and "Substrate type" = "sphagnum1."

$\$$ "Conditioned" refers to the variance partialled out by $\mathbf{V}_{K}$, "Constrained" refers to the (remaining) variance explained by predictors in $\mathbf{X}$, and "Unconstrained" refers to the residual variance.

$\S K_{\text {comm }}$ lists all significant component patterns at the community level, $K_{\mathrm{spp}}$ lists all component patterns that were significant for at least one species, and "No. species" indicates the number of species with at least one significant component pattern, based on a permutation test of model residuals with $\alpha=0.05$ and $R=999$ permutations.

- Moran's $I$ refers to spatial autocorrelation in the residuals (all values significant based on $\alpha=0.05$ and $R=999$ permutations).

autocorrelation in the residuals. Specifically, the single significant pattern $K=\{1\}$ in the original values Y (Fig. 4A) was explained by the two predictors, but the residuals showed two significant patterns for $K=\{2$,
4\} (permutation test with $R=999$ permutations; Fig. 4B).

In conditioned SCR with $K=\{2,4\}$ (Table 2: Model $3)$, the two patterns in $\mathbf{V}_{K}$ accounted for $7.3 \%$ of the
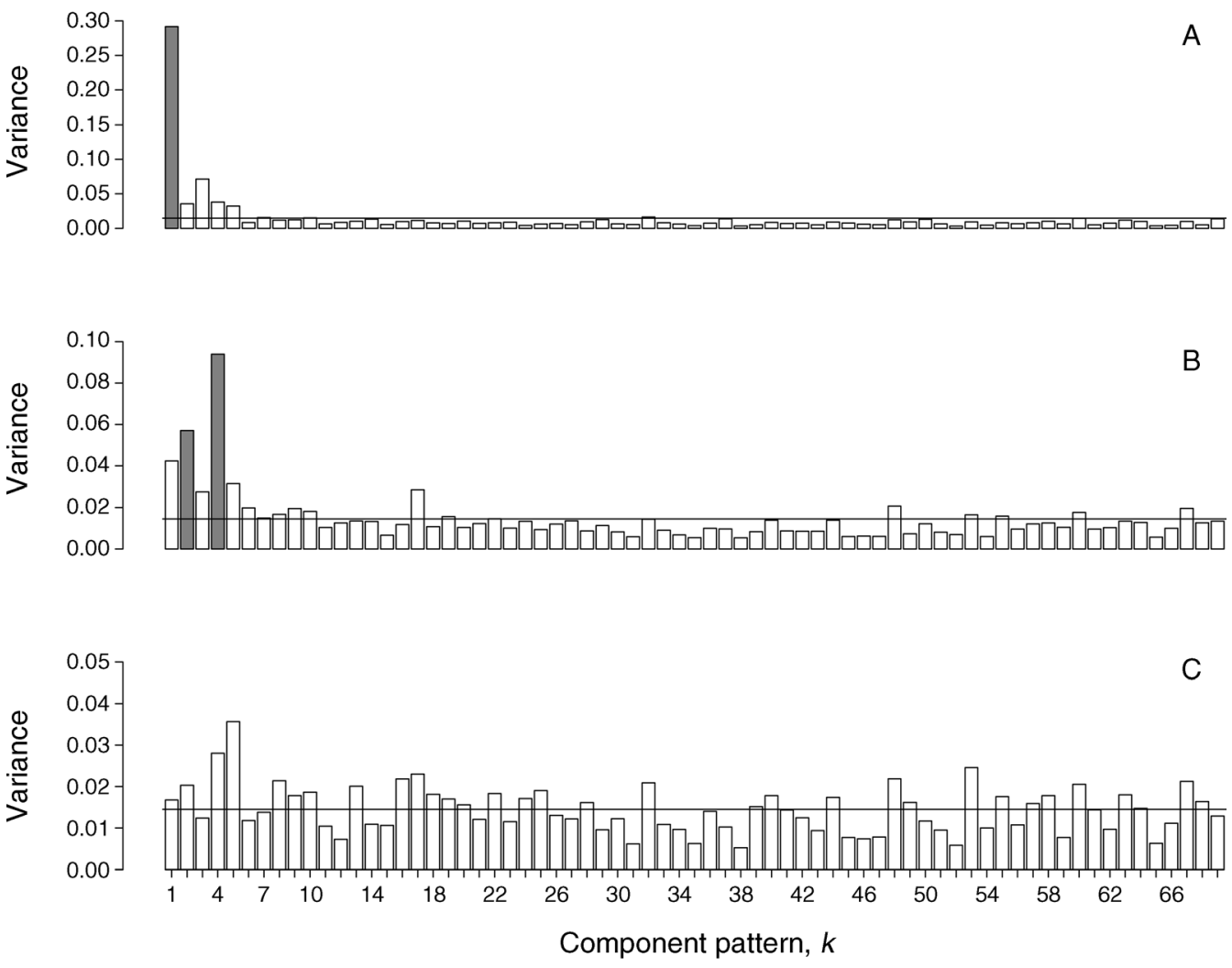

FIG. 4. Barplots of multivariate scalograms $\mathbf{s}$ of orbibatid mite data. Each bar shows the variance associated with one of the 69 component patterns in matrix $\mathbf{V}$ : (A) for the original response data $\mathbf{Y}$, (B) for the residuals of the initial model (with two predictors: substrate density and water content, and (C) for the residuals of the final model (with additional predictors shrub density, microtopography, and a dummy variable for substrate type = "sphagnum 1 ." The horizontal lines denote the expected value of $1 /(n-1)$. Bars with gray shading indicate significant component patterns (one-sided permutation test with $R=999, \alpha=0.05$ ). 
total variance in $\mathbf{Y}$ (conditioned variance), and the two predictors explained an additional $35.4 \%$ of variance after partialling out the patterns in $K$ (constrained variance) (Table 2: Model 3), which is more than in Model 2. The residuals showed no significant component patterns at the community level, though three species showed significant component patterns.

However, pattern $k=4$ was associated with factors shrub density and substrate type (especially "Sphagnum1"), and pattern $k=6$ was correlated with factor micro-topography (Table 3, Fig. 5). The final model (Table 2: Model 4), with the two quantitative predictors substrate density and water content, the two factors shrub density and microtopography, and a dummy variable for substrate type = "Sphagnum1," explained $46.0 \%$ of the variance of $\mathbf{Y}$. No single component pattern $k$ was statistically significant at the community level (Fig. 4C), whereas two species showed significant component patterns at relatively fine scales (Table 2).

\section{Implications for multivariate analysis of ecological communities}

As the oribatid mite data illustrated, spatial autocorrelation in the original response $\mathbf{Y}$, here $K=\{1\}$, may be explained by $\mathbf{X}$, so that the same component patterns are not significant in the residuals of a correctly specified regression model. On the other hand, the significant component patterns in the residuals $(K=\{2,4\})$ were not significant in the original response $\mathbf{Y}$. This illustrates that significant component patterns should be identified from model residuals.

Ecological community data are prone to spatial autocorrelation that cannot be explained by the predictors in $\mathbf{X}$, e.g., due to spatially limited dispersal or unmeasured ecological factors. The oribatid mite example illustrated that partialling out significant spatial patterns identified from the residuals may change the multivariate regression parameters and thus result, e.g., in a stronger association between predictors and response (Table 2). Hence testing and accounting for significant component patterns in the residuals of direct ordination should become standard practice.

Based on the data alone, we cannot identify whether significant component patterns in the residuals are caused by biotic processes such as dispersal or whether the model is mis-specified, e.g., by the omission of an important predictor. Short-range dispersal in the form of a simple diffusion process is likely associated with the first one or few component patterns, whereas a missing factor may create spatial patterns at any scale. While many authors recommend plotting regression residuals in space to visually identify potential missing factors, this approach remains subjective and is not practical for multispecies data. SCR provides an efficient and quantitative way to screen a potentially large set of additional data layers for factors that may explain the spatial structure in the residuals of direct ordination (Table 3, Fig. 5). If possible, identifying such missing
TABLE 3. Identification of potential missing factors for the oribatid mite data.

\begin{tabular}{lrr}
\hline \hline \multicolumn{1}{c}{ Factor } & $k=2$ & $k=4$ \\
\hline Shrub density & & \\
None $\dagger$ & 0.44 & 0.10 \\
Few $\dagger$ & -0.36 & 0.05 \\
Many & -0.04 & -0.15 \\
Micro-topography & & \\
Blanket $\dagger$ & -0.18 & 0.41 \\
Hummock & 0.18 & -0.41 \\
Substrate type & & -0.08 \\
Sphagnum1 $\dagger$ & 0.40 & 0.12 \\
Sphagnum2 & -0.25 & 0.14 \\
Sphagnum3 & 0.07 & 0.30 \\
Sphagnum4 & -0.13 & 0.10 \\
Litter & 0.13 & 0.03 \\
Barepeat & -0.04 & \\
Interface & -0.21 & \\
\hline
\end{tabular}

Notes: The table lists the point-biserial correlation between factor levels (coded as dummy variables) and the two significant component patterns of Model 2 (see Table 2). A "†" indicates dummy variables included in Model 4 (see maps in Fig. 5).

factors should be preferred over the use of component patterns as proxies.

Ordination has the potential to do more than summarize community structure, as it bridges between species- and community-level analysis. In the SCR framework, the matrices of species-level partial beta coefficients, scalograms (Jombart et al. 2009), and contributions to Moran's I could thus be developed into tools to identify joint and individual spatial patterns and responses to environmental factors, which may shed new light onto community structure.

\section{Conclusion}

The integration of linear regression with Moran's eigenvector map (MEM) in the framework of spatial component regression (SCR) provides the insight that all variation is spatially structured. Based on the spatial configuration of the sampling locations alone, any observed variable can be fully decomposed into a set of uncorrelated spatial patterns. This raises the question of what constitutes spatial independence, as it no longer can be defined as the absence of spatial structure. Rather, spatial (or temporal) independence can be characterized by the absence of excess variance at any scale and thus constitute "spatial white noise" (Lennon 2000).

MEM and related spatial eigenvector methods provide a powerful partitioning that we have not yet fully exploited, largely due to the concentration of efforts on using space as a predictor. The new framework of SCR presented here integrates regression analysis, MEM and spatial statistics through a spatial decomposition of the regression coefficients, and will help scientists understand many questions relating to methodological choices and behavior of methods in a new light. The new understanding will facilitate interpretation of existing 


$$
\begin{array}{ll} 
& \bullet(-0.04,0.04) \\
(-0.23,-0.13) & \bullet(0.04,0.13) \\
(-0.13,-0.04) & \bullet(0.13,0.23)
\end{array}
$$

Significant component patterns

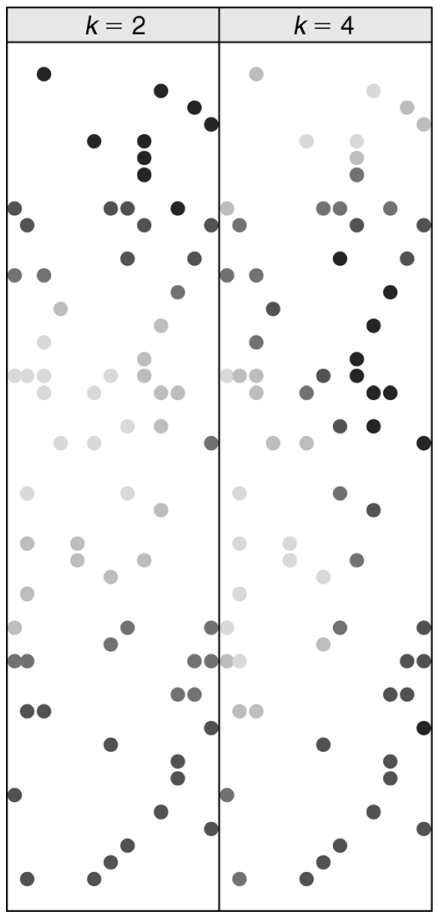

Four factor levels

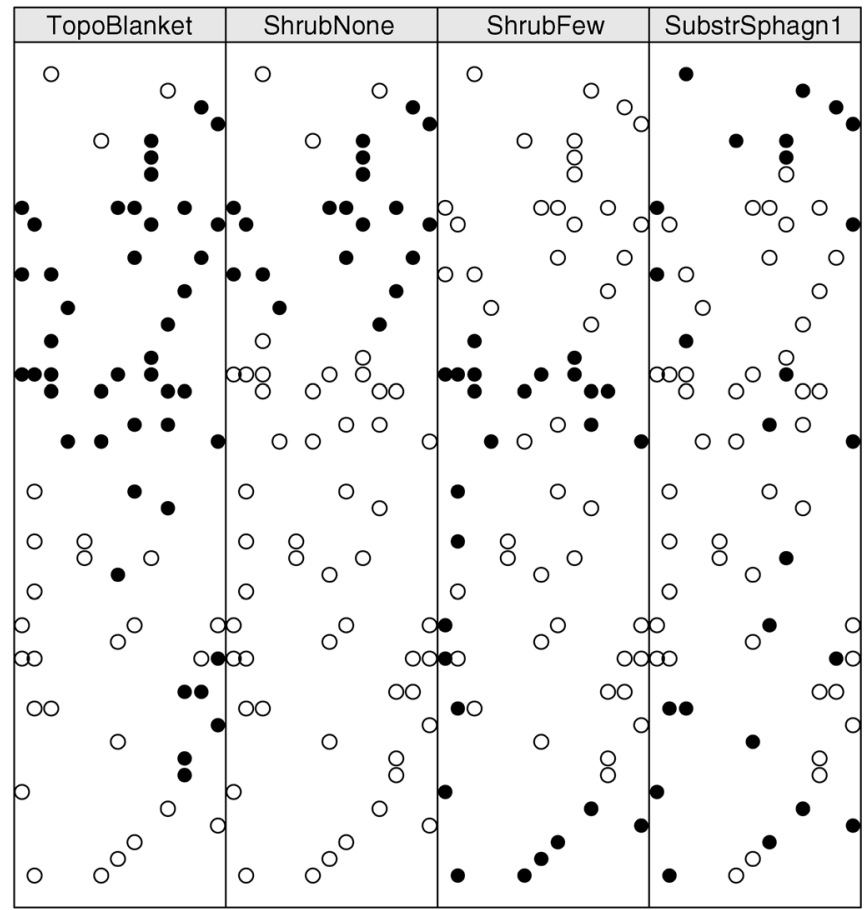

FIG. 5. Maps of (left) significant component patterns $K=\{2,4\}$ for the residuals of Model 2 and (right) four factor levels (coded as dummy variables) associated with these patterns (see Table 3). For significant component patterns, dark shades represent high scores and light shades low scores on spatial eigenvectors $k=2$ and $k=4$. For the factor levels TopoBlanket, ShrubNone, ShrubFew, and SubstSphagn1, open circles indicate that factor levels are not present, and solid black circles indicate presence of factor levels Mircro-topography $=$ Blanket, Shrub density $=$ None, Shrub density $=$ Few, and Substrate type $=$ Sphagnum 1 , respectively (see Table 3 ).

results and promote further methods development to address the complexity inherent in many ecological or evolutionary data sets. Sophisticated spatial-analysis tools will thus become available to practitioners in the familiar framework of the linear regression model.

\section{ACKNOWLEDGMENTS}

This research was supported through a Discovery Grant by the National Sciences and Engineering Council of Canada. I thank Marie-Josée Fortin, Erin Landguth, Shekhar Biswas, David Roberts, and J. A. F. Diniz-Filho for valuable comments on an earlier version of the manuscript.

\section{Literature Cited}

Anselin, L. 2002. Under the hood: Issues in the specification and interpretation of spatial regression models. Agricultural Economics 27:247-267.

Beale, C. M., J. J. Lennon, J. M. Yearsley, M. J. Brewer, and D. A. Elston. 2010. Regression analysis of spatial data. Ecology Letters 13:246-264.

Besag, J. 1974. Spatial interaction and the statistical analysis of lattice systems. Journal of the Royal Statistical Society Series B 36:192-236.

Bini, L. M., et al. 2009. Coefficient shifts in geographical ecology: an empirical evaluation of spatial and non-spatial regression. Ecography 32:193-204.
Bivand, R., et al. 2012. spdep: Spatial dependence: weighting schemes, statistics and models. $\mathrm{R}$ package version 0.5-45. http://CRAN.R-project.org/package=spdep

Blanchet, F. G., P. Legendre, and D. Borcard. 2008a. Forward selection of explanatory variables. Ecology 89:2623-2632.

Blanchet, F. G., P. Legendre, and D. Borcard. $2008 b$. Modelling directional spatial processes in ecological data. Ecological Modelling 215:325-336.

Borcard, D., and P. Legendre. 1994. Environmental control and spatial structure in ecological communities: an example using oribatid mites (Acari, Oribatei). Environmental and Ecological Statistics 1:37-61.

Borcard, D., and P. Legendre. 2002. All-scale spatial analysis of ecological data by means of principal coordinates of neighbour matrices. Ecological Modelling 153:51-68.

Borcard, D., P. Legendre, C. Avois-Jacquet, and H. Tuomisto. 2004. Dissecting the spatial structure of ecological data at multiple scales. Ecology 85:1826-1832.

Borcard, D., P. Legendre, and P. Drapeau. 1992. Partialling out the Spatial Component of Ecological Variation. Ecology 73: 1045-1055.

de Jong, P., C. Sprenger, and F. van Veen. 1984. On extreme values of Moran's I and Geary's c. Geographical Analysis 16: $17-24$

Diniz-Filho, J. A. F., and L. M. Bini. 2005. Modelling geographical patterns in species richness using eigenvectorbased spatial filters. Global Ecology and Biogeography 14: $177-185$. 
Diniz-Filho, J. A. F., L. M. Bini, and B. A. Hawkins. 2003. Spatial autocorrelation and red herrings in geographical ecology. Global Ecology and Biogeography 12:53-64.

Diniz-Filho, J. A. F., L. M. Bini, T. F. Rangel, I. MoralesCastilla, M. Á. Olalla-Tárraga, M. Á. Rodríguez, and B. A. Hawkins. 2012a. On the selection of phylogenetic eigenvectors for ecological analyses. Ecography 35:239-249.

Diniz-Filho, J. A. F., C. E. R. de Sant'Ana, and L. M. Bini. 1998. An eigenvector method for estimating phylogenetic inertia. Evolution 52:1247-1262.

Diniz-Filho, J. A. F., T. F. Rangel, T. Santos, and L. M. Bini. 2012b. Exploring patterns of interspecific variation in quantitative traits using sequential phylogenetic eigenvector regressions. Evolution 66:1079-1090.

Dormann, C. F., et al. 2007. Methods to account for spatial autocorrelation in the analysis of species distributional data: a review. Ecography 30:609-628.

Dray, S. 2011. A new perspective about Moran's coefficient: spatial autocorrelation as a linear regression problem. Geographical Analysis 43:127-141.

Dray, S., P. Legendre, and P. R. Peres-Neto. 2006. Spatial modelling: a comprehensive framework for principal coordinate analysis of neighbour matrices (PCNM). Ecological Modelling 196:483-493.

Fortin, M.-J., P. M. A. James, A. MacKenzie, S. J. Melles, and B. Rayfield. 2012. Spatial statistics, spatial regression, and graph theory in ecology. Spatial Statistics 1:100-109.

Griffith, D. A. 2000. A linear regression solution to the spatial autocorrelation problem. Journal of Geographical Systems 2: 141-156.

Griffith, D. A. 2005. Effective geographic sample size in the presence of spatial autocorrelation. Annals of the Association of American Geographers 95:740-760.

Griffith, D. A., and G. Arbia. 2010. Detecting negative spatial autocorrelation in georeferenced random variables. International Journal of Geographical Information Science 24:417437.

Griffith, D. A., and P. R. Peres-Neto. 2006. Spatial modeling in ecology: the flexibility of eigenfunction spatial analyses. Ecology 87:2603-2613.

Hawkins, B. A., and J. A. F. Diniz-Filho. 2004. "Latitude" and geographic patterns in species richness. Ecography 27:268272.
Jombart, T., S. Devillard, A. Dufour, and D. Pontier. 2008. Revealing cryptic spatial patterns in genetic variability by a new multivariate method. Heredity 101:92-103.

Jombart, T., S. Dray, and A. Dufour. 2009. Finding essential scales of spatial variation in ecological data: a multivariate approach. Ecography 32:161-168.

Legendre, P. 1993. Spatial autocorrelation: trouble or new paradigm? Ecology 74:1659-1673.

Legendre, P., and E. Gallagher. 2001. Ecologically meaningful transformations for ordination of species data. Oecologia 129:271-280.

Legendre, P., and L. Legendre. 2012. Numerical ecology. Elsevier, San Diego, California, USA.

Lennon, J. J. 2000. Red-shifts and red herrings in geographical ecology. Ecography 23:101-113.

Moran, P. A. P. 1950. Notes on continuous stochastic phenomena. Biometrika 37:17-23.

Oksanen, J., F. G. Blanchet, R. Kindt, P. Legendre, P. R. Minchin, R. B. O'Hara, G. L. Simpson, P. Solymos, M. H. H. Stevens, and H. H. Wagner. 2012. vegan: Community Ecology Package. $\mathrm{R}$ package version 2.0-5. http://CRAN.R-project.org/package=vegan

Patuelli, R., D. Griffith, M. Tiefelsdorf, and P. Nijkamp. 2011. Spatial filtering and eigenvector stability: space-time models for German unemployment data. International Regional Science Review 34:253-280.

R Development Core Team. 2012. R: a language and environment for statistical computing. R Foundation for Statistical Computing, Vienna, Austria. http://www. R-project.org/

Rohlf, F. J. 2001. Comparative methods for the analysis of continuous variables: geometric interpretations. Evolution 55:2143-2160.

Tiefelsdorf, M., and A. G. Daniel. 2007. Semiparametric filtering of spatial autocorrelation: the eigenvector approach. Environment and Planning A 39:1193-1221.

Wagner, H. H., and M. J. Fortin. 2005. Spatial analysis of landscapes: concepts and statistics. Ecology 86:1975-1987.

Whittle, P. 1954. On stationary processes in the plane. Biometrika 41:434-449.

Wiens, J. A. 1989. Spatial scaling in ecology. Functional Ecology 3:385-397.

\section{Supplemental Material}

Supplement 1

$\mathrm{R}$ code for analysis of simulated data (Ecological Archives E094-219-S1).

Supplement 2

$\mathrm{R}$ code for analysis of oribatid mite data (Ecological Archives E094-219-S2). 